\title{
Robótica na cirurgia odontológica: Revisão integrativa
}

\author{
Robotics in oral surgical procedures: Integrative review
}

Robótica en procedimientos quirúrgicos orales: Revisión integrativa

Isis Samara de Melo Queiroga ORCID: https://orcid.org/0000-0002-9434-5741 Faculdade de Odontologia de Pernambuco, Brasil E-mail: dulceeisis@gmail.com

Maria Clara de Aquino Veras Falcão

ORCID: https://orcid.org/0000-0002-8829-1310 Faculdade de Odontologia de Pernambuco, Brasil E-mail: clara.aquino.v.f@gmail.com

Esdras Gabriel Alves-Silva

ORCID: https://orcid.org/0000-0002-2309-1115 Universidade Estadual de Campinas, Brasil E-mail: esdras0702@yahoo.com.br Eloiza Leonardo de Melo

ORCID: https://orcid.org/0000-0001-5845-1478 Faculdade de Odontologia de Pernambuco, Brasil E-mail: eloiza.leonardo@upe.br

Marleny Elizabeth Márquez de Martínez Gerbi

ORCID: https://orcid.org/0000-0001-9174-2541 Faculdade de Odontologia de Pernambuco, Brasil E-mail: marleny.gerbi@upe.br

Mávio Eduardo Azevedo Bispo

ORCID: https://orcid.org/0000-0002-3781-0272 Escola Politécnica de Pernambuco, Brasil E-mail: meb@ecomp.upe.br

Renata Araújo Gomes de Sá

ORCID: https://orcid.org/0000-0001-6550-8225 Faculdade de Odontologia de Pernambuco, Brasil E-mail: renata.agsa@gmail.com

Maria Regina Almeida de Menezes

ORCID: https://orcid.org/0000-0003-3012-3979

Faculdade de Odontologia de Pernambuco, Brasil E-mail: regina.menezes@upe.br

\begin{abstract}
Resumo
Os robôs são uma inovação tecnológica adaptada para utilização em procedimentos cirúrgicos dos mais variados âmbitos, auxiliando o profissional de saúde no cumprimento de seu papel. Quando associados à odontologia, tem-se aplicabilidade em diversas áreas como a oncologia e implantodontia. O objetivo deste trabalho foi realizar uma revisão integrativa da literatura atual sobre procedimentos cirúrgicos robotizados na odontologia, destacando suas técnicas, indicações, vantagens e desvantagens. Foi feita uma pesquisa dos artigos nas bases de dados eletrônicas CAPES, PubMed e MEDLINE, utilizando os descritores: "Robotic Surgical" "Procedures", "Dentistry", "Oral Surgery", indexados no período de 2015 a 2020, que tratavam de relatos de caso, revisões de literatura, estudos in vivo que relatam a utilização de robôs em procedimentos cirúrgicos odontológicos, estudos de viabilidade e ensaios clínicos. Após os critérios de elegibilidade, foram analisados 18 artigos integralmente publicados em língua inglesa. Os estudos mostraram que a utilização de robôs na cirurgia odontológica traz mais precisão ao procedimento, diminuindo a perda de tecido, reduzindo a possibilidade de hemorragia, reduzindo possíveis cicatrizes resquiciais, e pós-cirúrgico. A cirurgia robótica em diversos âmbitos da odontologia já demonstrou alta confiabilidade permitindo desde melhor precisão até maior assertividade, além de proporcionar um acesso cirúrgico menos invasivo com dano tecidual adjacente à área de atuação relativamente menor, otimizando o procedimento cirúrgico.
\end{abstract}

Palavras-chave: Procedimentos cirúrgicos robóticos; Odontologia; Cirurgia oral.

\section{Abstract}

Robots are a technological innovation adapted to use in surgical procedures of the most varied areas, assisting health professionals in fulfilling their role. When associated with dentistry, they have applicability in several areas such as oncology and implantology. The objective of this work was to carry out an integrative review of the current literature on robotic surgical procedures in dentistry, highlighting its techniques, indications, advantages and disadvantages. A 
research of articles had been done on the electronic databases CAPES, PubMed and MEDLINE, using the descriptors: robotic surgical procedures, dentistry, oral surgery, indexed in the period from 2015 to 2020 that dealt with case reports, literature reviews, in vivo studies that report the use of robots in dental surgical procedures, feasibility studies and clinical trials. After the eligibility criteria, 18 articles published in English were analyzed. Studies have shown that the use of robots in dental surgery brings more precision to the procedure, decreasing tissue loss, reducing the possibility of hemorrhage, reducing possible residual scarring, and post-surgical. Robotic surgery in several areas of dentistry has already demonstrated high reliability, allowing from better precision to greater assertiveness, in addition to providing less invasive surgical access with damage tissue adjacent to the relatively smaller area of operation, optimizing the surgical procedure.

Keywords: Robotic surgical procedures; Dentistry; Oral surgery.

\section{Resumen}

Los robots son una innovación tecnológica adaptada para la utilización en procedimientos quirúrgicos de los más variados ámbitos, asistiendo al profesional de la salud en el cumplimiento de su papel. Asociándose a la odontología, se encuentran aplicabilidades en diversas áreas como la oncología e implantología dental. El objetivo de este trabajo fue realizar una revisión integrativa de la literatura actual sobre procedimientos quirúrgicos robotizados en la odontología, destacando sus técnicas, indicaciones, ventajas y desventajas. Fue hecha una investigación de los artículos en las bases de datos electrónicas CAPES, PubMed y MEDLINE, utilizando los descriptores: "Robotic Surgical" "Procedures", "Dentistry", "Oral Surgery", indexados en el periodo de 2015 a 2020, que trataban de reporte de caso, revisiones de la literatura, estudios in vivo que relatan la utilización de robots en procedimientos quirúrgicos, odontológicos, estudios de viabilidad y ensayos clínicos. Posteriormente a los criterios de elegibilidad, fueron analizados 18 artículos integralmente publicados en lengua inglesa. Los estudios mostraron que la utilización de robots en la cirugía odontológica trae más precisión al procedimiento, disminuyendo la pérdida de tejido, reduciendo la posibilidad de hemorragia, reduciendo posibles resquicios de cicatrices, y post cirugía. La cirugía robótica en diversos ámbitos de la odontología ya demostró alta confiabilidad permitiendo desde mejor precisión hasta mayor asertividad, además de proporcionar un acceso cirúrgico menos invasivo con daño tisular adyacente al área de actuación relativamente menor, optimizando el procedimiento quirúrgico.

Palabras clave: Procedimientos quirúrgicos robóticos; Odontología; Cirugía oral.

\section{Introdução}

A utilização da robótica na área saúde pode ser observada desde meados dos anos 80. Atualmente robôs trazem a realidade dos avanços tecnológicos para a odontologia. Contudo, tal aplicação ainda é pouco disseminada nessa área, apesar de ser uma técnica bastante promissora no que diz respeito à praticidade e segurança, tanto para o cirurgião-dentista quanto para o paciente.

Apesar das atuais técnicas de cirurgias orais já apresentarem bons resultados, podem ser aprimoradas caso ocorra uma associação com as tecnologias adequadas para cada caso. O fato dos estudos sobre esses tipos de procedimentos serem recentes, deixam dúvidas quanto à rentabilidade, às suas vantagens e desvantagens ou à possibilidade de aplicação.

Com a necessidade de precisão, tem-se uma das maiores vantagens das cirurgias robotizados, pois a exatidão operatória reduz sangramentos e dores na fase de recuperação, que costuma ser mais rápida. Com isso, podem ser utilizados em cirurgias orais de alto grau de complexidade e ainda possibilitam a redução no número de infecções hospitalares.

Nos casos em que as cirurgias robotizadas são indicadas, deve ser levado em conta se esse tipo de procedimento produzirá melhores resultados que o convencional e qual o tipo de abordagem deve ser feita em cada caso. Com isso, a aplicação dessa nova técnica de realização das cirurgias ocorre de uma forma mais segura, prática e que proporciona melhores resultados. Esse método pode ser aplicado em várias vertentes, como a cirurgia transoral robótica (TORS), na oncologia, e a perfuração definida de orifícios ou leitos de implante com parada automática na implantodontia, conferindo diversas vantagens quando comparado ao método tradicional.

Dessa forma, o objetivo do presente estudo é realizar uma revisão integrativa da literatura para investigar a eficácia da robótica na realização de procedimentos cirúrgicos odontológicos, destacando suas indicações, vantagens e desvantagens. 


\section{Metodologia}

\subsection{Estratégia de Busca}

O presente estudo trata-se de uma revisão integrativa da literatura, executada por meio de uma pesquisa de artigos científicos nas bases de dados eletrônicos PubMed, Medline e Capes fazendo uso dos descritores "Robotic Surgical Procedures AND Dentistry AND Oral, surgery" estabelecidos com as associações e combinações de interesse dos autores, foram elaborados, entre os descritores, as seguintes pesquisas ilustradas na Figura 1.

Figura 1. Fluxograma de estratégia de busca nas bases de dados.

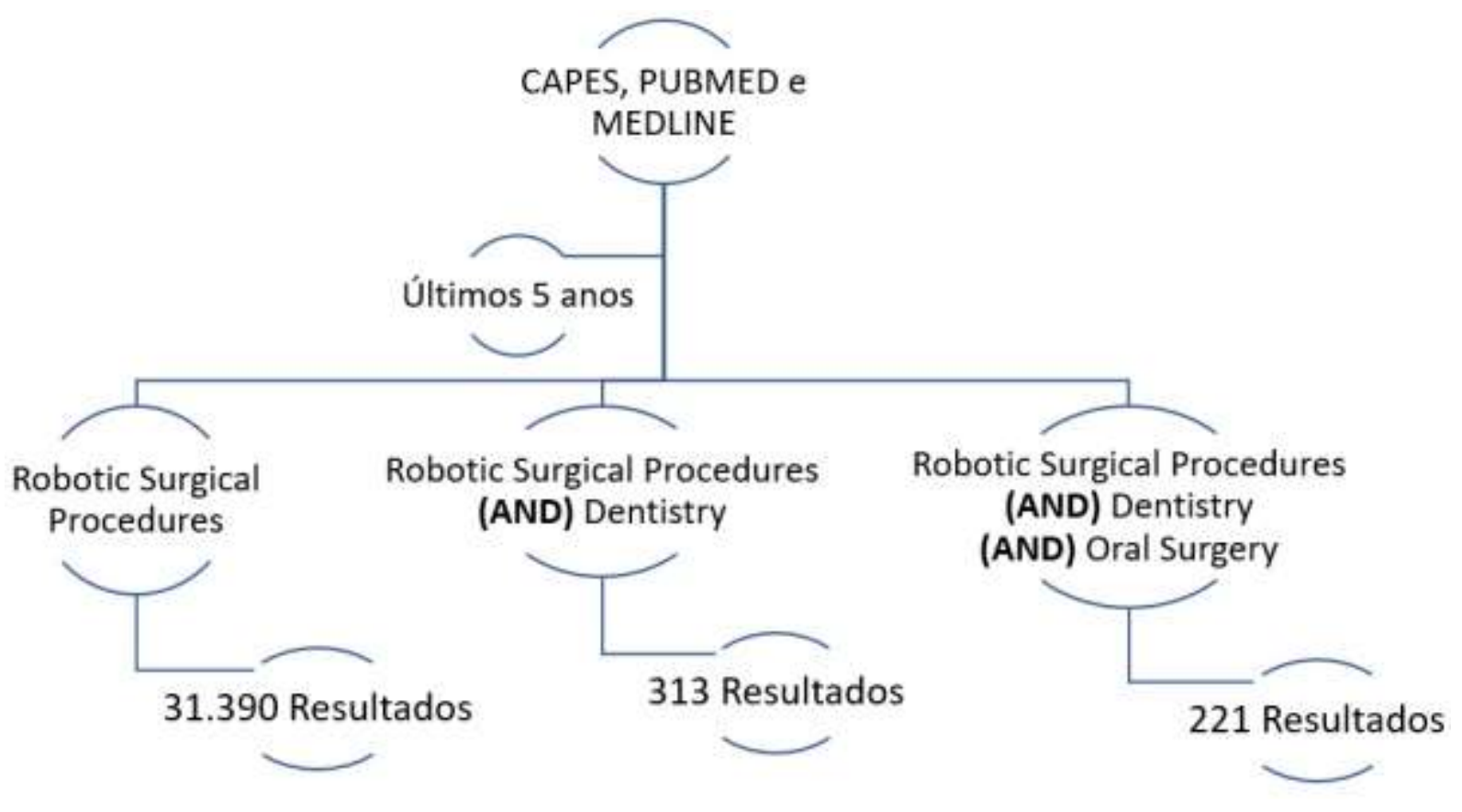

Fonte: Autores (2020).

A partir da busca de artigos científicos nas plataformas acima evidenciadas, no período de 4 a 13 agosto de 2020, utilizando o descritor "Robotic Surgical Procedures", obtivemos um total de 31.390 artigos, após a junção desse descritor com "Dentistry" o resultado foi de 312 artigos. Foram filtrados os artigos já encontrados na pesquisa anterior com apenas o primeiro descritor, então foi feita a junção dos descritores anteriores mais "Oral, surgery" e obtivemos um total de 221 artigos, sem haver repetição deles em nenhum banco de dados.

O processo de seleção para avaliação e escolha dos artigos foi realizado por duas pesquisadoras, de forma independente, com uma subsequente comparação dos resultados para obtenção dos elementos selecionados em comum acordo. Os elementos listados repetidamente nos dois bancos de dados foram considerados uma única vez. Então, a seleção das publicações foi conduzida em duas etapas: (1) leitura dos resumos dos artigos e (2) análise qualitativa dos artigos na íntegra.

Para a escolha das fontes, foram consideradas como critérios de inserção dos artigos originais, publicados em língua inglesa e indexados no período de 2015 a agosto de 2020, que se tratavam de artigos de revisão integrativa, relato de casos, estudos in vivo que relatassem a utilização de robôs em procedimentos cirúrgicos na odontologia, estudos de viabilidade e ensaios clínicos.

Não foram incluídos artigos de revisão de literatura nem relatos de casos publicados em outros idiomas ou em um intervalo de tempo diferente do determinado. A Figura 2 ilustra a estratégia de busca de acordo com os critérios citados. 
Figura 2. Fluxograma de estratégia de busca e seleção dos artigos.

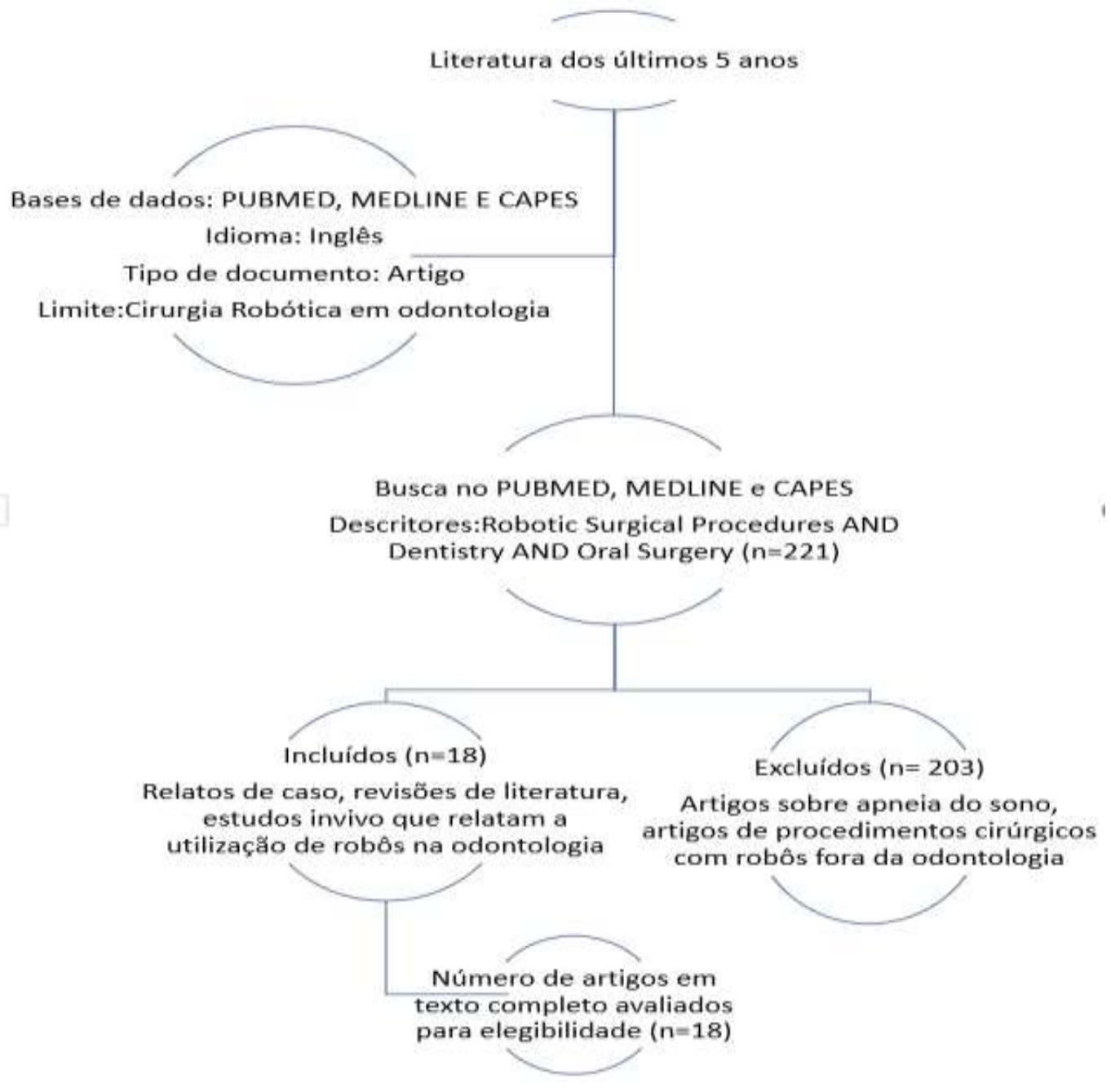

Fonte: Autores (2020).

Esta metodologia seguiu as recomendações PRISMA (Principais Itens para Relatar Revisões Sistemáticas e Metanálise), trata-se de uma revisão integrativa de pesquisa qualitativa. Possibilitando aos autores a exploração das vantagens, das limitações, da relevância e do impacto do uso de robôs em cirurgias odontológicas. Os artigos incompatíveis nos critérios de inclusão, ou repetidos, foram excluídos. Em seguida, dos artigos restantes, foi realizada a leitura de forma integral, chegando a quantidade final de artigos incluídos nesta revisão.

Não foram utilizadas teses, dissertações e monografia, em razão que a realização de uma busca desses estudos é inexecutável logisticamente e as bases de dados utilizadas na pesquisa não possuem esse tipo de publicação.

\section{Resultados e Discussão}

\subsection{Resultados}

\subsubsection{Objetivo}

O presente estudo teve como objetivo analisar a utilização de robôs em procedimentos cirúrgicos odontológicos, observando as vantagens, desvantagens e eficácia desse uso em intervenções. A revisão integrativa obteve estudos recentes que trazem a criação dessa técnica, o seu desenvolvimento e os avanços, mostrando os diversos casos em que robôs podem auxiliar ou realizar intervenções cirúrgicas de pequeno, médio e grande porte, como mostra na Quadro 1. 
Quadro 1. Seleção dos artigos de acordo com o autor, tipo de estudo, objetivos, metodologia, resultados e conclusão.

\begin{tabular}{|c|c|c|c|c|}
\hline $\begin{array}{l}\text { Autor e } \\
\text { Estudo }\end{array}$ & Objetivo & Metodologia & Resultados & Conclusão \\
\hline $\begin{array}{l}\text { GULATI, } \\
\text { M. et al. } \\
2015\end{array}$ & $\begin{array}{l}\text { Demonstrar os avanços da } \\
\text { informatização } \\
\text { implantodontia e } \\
\text { benefícios peus } \\
\text { pacientes. }\end{array}$ & $\begin{array}{l}\text { Para superar essas limitações da imagem } \\
\text { bidimensional e imprecisões na fabricação do } \\
\text { stent ou canais guia, que muitas vezes levam à } \\
\text { colocação incorreta do implante, o que resulta em } \\
\text { complicações e falha do implante, especialmente } \\
\text { em situações anatomicamente complicadas, foram } \\
\text { feitos muitos avanços, que informatizaram a } \\
\text { implantodontia. }\end{array}$ & $\begin{array}{l}\text { Os robôs facilitam os procedimentos } \\
\text { cirúrgicos por serem mais precisos e } \\
\text { confiáveis que os seres humanos, são } \\
\text { minimamente invasivos, resultam em uma } \\
\text { recuperação mais rápida comparada a uma } \\
\text { cirurgia tradicional, são imunes à radiação, } \\
\text { reduzem o tempo da cirurgia e acarretam } \\
\text { menos danos às estruturas da área a ser } \\
\text { operada. }\end{array}$ & $\begin{array}{l}\text { A automação da implantodontia permitiu } \\
\text { que os implantes fosses realizados até } \\
\text { mesmo em pacientes que antes a cirurgia } \\
\text { era contraindicada. Os avanços } \\
\text { tecnológicos nessa área permitirão } \\
\text { imagens intraoperatórias avançadas e } \\
\text { ferramentas robóticas sofisticadas que } \\
\text { facilitarão ainda mais os procedimentos } \\
\text { cirúrgicos e a recuperação do paciente. }\end{array}$ \\
\hline $\begin{array}{l}\text { REDDY, } \\
\text { E. D. et } \\
\text { al. } \\
2015\end{array}$ & $\begin{array}{l}\text { Demonstrar que o uso da } \\
\text { técnica de radiocirurgia } \\
\text { estereotáxica com o } \\
\text { CybeKnife pode ser uma } \\
\text { alternativa viável em } \\
\text { relação ao tratamento } \\
\text { cirúrgico convencional do } \\
\text { câncer. }\end{array}$ & $\begin{array}{l}\text { Em 2014, Yamazaki et al. realizou um estudo no } \\
\text { qual os pacientes com câncer ( } 11 \text { de nasofaringe, } \\
7 \text { de orofaringe, } 1 \text { de hipofaringe, } 3 \text { de cavidade } \\
\text { nasal e de seios paranasais, e } 3 \text { de cânceres orais } \\
\text { já tratados com radioterapia convencional) e fez o } \\
\text { tratamento com CyberKnife, } 18 \text { dos pacientes } \\
\text { mostraram resposta completa, } 6 \text { mostraram } \\
\text { resposta parcial e } 1 \text { teve doença progressiva. } \\
\text { CyberKnife provou ser um excelente } \\
\text { procedimento no tratamento da neuralgia do } \\
\text { trigêmeo em uma revisão de } 10 \text { estudos relatados } \\
\text { por Gibbs et al. em } 2009 \text {, em que } 77-96 \% \text { dos } \\
\text { pacientes relataram alívio inicial da dor e } 4,8-34 \% \\
\text { relatou recorrência da dor. }\end{array}$ & $\begin{array}{l}\text { O instrumento com maior } \mathrm{O} \text { uso do } \\
\text { Cyberknife é o tratamento escolhido em } \\
\text { casos nos quais novas intervenções } \\
\text { cirúrgicas não são viáveis, como por } \\
\text { exemplo lesões inacessíveis ou localizadas } \\
\text { próximo a estruturas críticas e tumores } \\
\text { recorrentes ou residuais, onde o tratamento } \\
\text { de radioterapia anterior já foi realizado. O } \\
\text { seu uso requer uma equipe maior que o } \\
\text { convencional e mais preparada, além de ter } \\
\text { um tempo maior de tratamento o que pode } \\
\text { levar à limitação quanto ao seu uso. }\end{array}$ & $\begin{array}{l}\text { A incisão mais regular foi } \mathrm{O} \text { uso da } \\
\text { robótica na rádio cirurgia, especificamente } \\
\text { o sistema CyberKnife provou ser } \\
\text { altamente preciso, seguro e indolor. É } \\
\text { indicado na odontologia como linha de } \\
\text { tratamento de câncer de cabeça e pescoço e } \\
\text { neuralgia do trigêmeo. }\end{array}$ \\
\hline
\end{tabular}




\begin{tabular}{|c|c|c|c|c|}
\hline $\begin{array}{l}\text { Mazeroll } \\
\text { e, P. et al. } \\
2018\end{array}$ & $\begin{array}{l}\text { Apresentar os resultados } \\
\text { oncológicos e funcionais } \\
\text { da TORS para CCE de } \\
\text { seio piriforme. }\end{array}$ & $\begin{array}{l}\text { Foram incluídos, retrospectivamente, todos os } \\
\text { procedimentos TORS para carcinoma de células } \\
\text { escamosas (CEE) de seio piriforme realizados } \\
\text { entre } 2009 \text { e } 2017 \text { em oito centros de referência } \\
\text { terciários franceses. Foram excluídas as lesões } \\
\text { envolvendo os seios piriformes que se } \\
\text { desenvolveram da orofaringe, laringe ou outros } \\
\text { sub sítios anatômicos da hipofaringe. }\end{array}$ & $\begin{array}{l}\text { Foram incluídos } 57 \text { procedimentos TORS. } \\
\text { A permanência hospitalar média foi de } 10 \\
\text { dias. Após um acompanhamento médio de } \\
23 \text { meses, sobrevida geral e livre de } \\
\text { doença foram, respectivamente, } 84 \% \text { e } \\
74 \% \text { em } 24 \text { meses, e } 66 \% \text { e } 50 \% \text { em } 48 \\
\text { meses. Ao final do acompanhamento, a } \\
\text { taxa de preservação do órgão foi de } 96 \% \text {. } \\
\text { Nenhum dos pacientes sobreviventes } \\
\text { necessitaram de traqueotomia e a dieta oral } \\
\text { foi possível para } 96 \% \text {. }\end{array}$ & $\begin{array}{l}\text { TORS pode ser considerado um } \\
\text { procedimento conservador e seguro para } \\
\text { pequenas lesões no seio piriforme, na } \\
\text { primeira terapia ou na terapia de salvação. } \\
\text { Tem resultados oncológicos aceitáveis e } \\
\text { excelentes resultados funcionais. No } \\
\text { entanto, é um dos tipos mais complexos de } \\
\text { cirurgia assistida por robô e a estrita } \\
\text { seleção de casos é necessária, } \\
\text { principalmente para lesões que envolvem o } \\
\text { ângulo anterior. }\end{array}$ \\
\hline $\begin{array}{l}\text { Ling, D. } \\
\text { C. et al. } \\
2016\end{array}$ & $\begin{array}{l}\text { Comparar os resultados } \\
\text { oncológicos e de } \\
\text { qualidade de vida (QV) } \\
\text { entre a TRC definitiva e a } \\
\text { TORS definitiva. }\end{array}$ & $\begin{array}{l}\text { Um estudo comparativo observacional foi } \\
\text { realizado em } 92 \text { pacientes tratados com TORS } \pm \\
\text { terapia adjuvante e } 46 \text { pacientes tratados com } \\
\text { CRT definitiva entre julho de } 2005 \text { e janeiro } \\
2016 \text {. O método Kaplan Meier foi usado para } \\
\text { análises de sobrevivência, e o teste de Mann- } \\
\text { Whitney foi usado para comparar os escores de } \\
\text { QV entre os grupos. }\end{array}$ & $\begin{array}{l}\text { Todos os pacientes tinham doença T0-T2 e } \\
\text { N0-N2, embora os pacientes com TRC } \\
\text { apresentassem estadiamento clínico mais } \\
\text { elevado. A doença HPV + estava presente } \\
\text { em } 79 \%(n=73) \text { dos pacientes com TORS } \\
\text { e } 91 \%(n=19) \text { dos CRT testados. O } \\
\text { acompanhamento médio foi de } 22,1 \text { meses. } \\
\text { Não houve diferenças significativas no } \\
\text { controle locorregional ou sobrevida global } \\
\text { entre os grupos CRT e TORS. }\end{array}$ & $\begin{array}{l}\text { CRT definitivo e TORS definitivo } \\
\text { oferecem taxas semelhantes de controle } \\
\text { loco-regional, sobrevida global, e } \\
\text { sobrevida livre de doença em pacientes } \\
\text { com CEE em estágio inicial. TORS } \\
\text { resultou em um curto significativamente } \\
\text { melhor e QV relacionada à saliva em } \\
\text { longo prazo, enquanto a terapia adjuvante } \\
\text { foi associada a pior saliva e QV } \\
\text { relacionada ao sabor em comparação com } \\
\text { TORS sozinho. }\end{array}$ \\
\hline $\begin{array}{l}\text { Liu, H. et } \\
\text { al. } \\
2019\end{array}$ & $\begin{array}{l}\text { Comparar a eficácia e } \\
\text { segurança da cirurgia } \\
\text { robótica com a de } \\
\text { operações abertas para } \\
\text { pacientes com câncer de }\end{array}$ & $\begin{array}{l}\text { Foram } 1731 \text { documentos recuperados durante a } \\
\text { pesquisa e, após removidas duplicatas, } 818 \text { foram } \\
\text { deixados, incluindo } 10 \text { em andamento estudos e } \\
\text { artigos cinzentos. Após a triagem do texto } \\
\text { completo, } 13 \text { artigos foram incluídos na revisão. }\end{array}$ & $\begin{array}{l}\text { Treze estudos que compararam a ressecção } \\
\text { robótica da parte superior via aérea e trato } \\
\text { digestivo por uma abordagem transoral } \\
\text { com abertura ressecção por abordagem } \\
\text { cervical ou transmandibulotomia }\end{array}$ & $\begin{array}{l}\text { O sistema cirúrgico robótico tem } \\
\text { benefícios notáveis na redução da taxa de } \\
\text { margens invadidas, diminuindo as } \\
\text { complicações e melhorando a qualidade de } \\
\text { vida dos pacientes em comparação com a }\end{array}$ \\
\hline
\end{tabular}




\begin{tabular}{|c|c|c|c|c|}
\hline & cabeça e pescoço. & & $\begin{array}{l}\text { preencheram os critérios de inclusão e } \\
\text { exclusão e foram incluídos nesta revisão. }\end{array}$ & $\begin{array}{l}\text { cirurgia aberta convencional para câncer } \\
\text { de cabeça e pescoço. O seu alto custo pode } \\
\text { ser a principal limitação até agora. }\end{array}$ \\
\hline $\begin{array}{l}\text { Kumar, } \\
\text { A. et al. } \\
2016\end{array}$ & $\begin{array}{l}\text { Demonstrar como o } \\
\text { cirurgião-dentista pode } \\
\text { realizar uma cirurgia } \\
\text { através do endoscópio e } \\
\text { orientar considerando as } \\
\text { manobras cirúrgicas no } \\
\text { monitor, sem obstáculos } \\
\text { para a observação. }\end{array}$ & $\begin{array}{l}\text { A cirurgia endoscopicamente assistida está se } \\
\text { tornando uma ferramenta comumente aplicada } \\
\text { para auxiliar e simplificar um pouco vários } \\
\text { procedimentos complicados que muitas vezes } \\
\text { necessitam de uma exposição cirúrgica mais } \\
\text { abrangente para visualização. Ao permitir que os } \\
\text { cirurgiões operem de dentro da boca do paciente, } \\
\text { evitando incisões externas no pescoço e na } \\
\text { mandíbula que podem deixar um indivíduo com } \\
\text { cicatrizes persistentes e complicações para comer, } \\
\text { falar e engolir. }\end{array}$ & $\begin{array}{l}\text { A tecnologia de robô minimamente } \\
\text { invasiva foi inventada para dar aos } \\
\text { cirurgiões habilidades aprimoradas, } \\
\text { abrangendo visualização, precisão e } \\
\text { controle inovadores. O sistema robótico } \\
\text { atua transformando os movimentos das } \\
\text { mãos do cirurgião no mecanismo de } \\
\text { controle robótico em movimentos muito } \\
\text { mais precisos dos instrumentos } \\
\text { miniaturizados que são injetados no corpo. }\end{array}$ & $\begin{array}{l}\text { A supervisão endoscopicamente assistida } \\
\text { de trauma maxilofacial tem sido relatada } \\
\text { como o tratamento de fraturas do côndilo } \\
\text { mandibular, fraturas do complexo } \\
\text { zigomático, fraturas orbitárias e fraturas do } \\
\text { seio frontal. Embora o tratamento cirúrgico } \\
\text { para redução aberta e fixação de fraturas } \\
\text { por osteossíntese de miniplaca não tenha } \\
\text { sido substituído, os procedimentos } \\
\text { endoscopicamente assistidos tornam } \\
\text { desejável limitar as incisões e aumentar o } \\
\text { controle intraoperatório sobre a redução da } \\
\text { fratura em regiões de exposição e } \\
\text { visibilidade restritas. }\end{array}$ \\
\hline $\begin{array}{l}\text { Sun, T. et } \\
\text { al. } \\
2020\end{array}$ & $\begin{array}{l}\text { Este estudo visa } \\
\text { principalmente } \\
\text { compreender a precisão do } \\
\text { mesmo dentista sob } \\
\text { diferentes sistemas } \\
\text { auxiliares. }\end{array}$ & $\begin{array}{l}\text { Os participantes tinham } 20 \text { anos ou mais e } \\
\text { precisavam de cirurgia de implante dentário. } 128 \\
\text { implantes dentários foram inseridos, } 32 \text { implantes } \\
\text { dentários em cada grupo e participantes com } \\
\text { idade semelhante ou idêntica, e os dentes faltantes } \\
\text { foram pareados para comparação. A precisão foi } \\
\text { medida pela sobreposição da posição real na } \\
\text { tomografia computadorizada de feixe cônico pós- } \\
\text { operatória com a colocação pré-cirúrgica virtual } \\
\text { do implante na imagem. }\end{array}$ & $\begin{array}{l}\text { Uma comparação dos métodos cirúrgicos } \\
\text { de implante dentário indicou que a } \\
\text { combinação de um sistema de navegação } \\
\text { de implante dentário e um kit de guia } \\
\text { cirúrgico atingiu a maior precisão em } \\
\text { termos das diferentes posições dos dentes e } \\
\text { mandíbulas. }\end{array}$ & $\begin{array}{l}\text { A precisão do sistema de navegação dental } \\
\text { sozinho é superior ao stent cirúrgico } \\
\text { sozinho e à mão livre cirurgia sozinho. } \\
\text { Uma comparação dos quatro métodos } \\
\text { cirúrgicos de implante dentário indicou } \\
\text { que a combinação do sistema de } \\
\text { navegação de implante dentário e kit guia } \\
\text { cirúrgico alcançaram a maior precisão em } \\
\text { termos das posições dos dentes e maxilares } \\
\text { superior e inferior. }\end{array}$ \\
\hline
\end{tabular}




\begin{tabular}{|c|c|c|c|c|}
\hline $\begin{array}{l}\text { Alfouzan, } \\
\text { A. F. et } \\
\text { al. } \\
2018\end{array}$ & $\begin{array}{l}\text { Esta revisão resume o } \\
\text { desenvolvimento da } \\
\text { ressecção e reconstrução } \\
\text { do câncer de cabeça e } \\
\text { pescoço. }\end{array}$ & $\begin{array}{l}\text { O câncer de cabeça e pescoço representa a sexta } \\
\text { neoplasia maligna mais frequente. Em todo o } \\
\text { mundo, mais de } 500.000 \text { novos casos são } \\
\text { diagnosticados anualmente. A ressecção cirúrgica } \\
\text { é a modalidade de tratamento primária para o } \\
\text { câncer oral. A ressecção do tumor envolve a } \\
\text { remoção da lesão com uma ampla margem de } \\
\text { tecido normal; linfonodos cervicais envolvidos } \\
\text { também precisam ser removidos. }\end{array}$ & $\begin{array}{l}\text { Nas últimas décadas, a cirurgia de } \\
\text { ressecção e reconstrução para câncer de } \\
\text { cabeça e pescoço desenvolveu-se } \\
\text { significativamente. Simulação cirúrgica, } \\
\text { impressão 3D, cirurgia microvascular e } \\
\text { cirurgia minimamente invasiva } \\
\text { melhoraram os resultados cirúrgicos e } \\
\text { levaram a resultados funcionais e estéticos } \\
\text { superiores. }\end{array}$ & $\begin{array}{l}\text { O planejamento de implantes dentários e } \\
\text { do tipo de prótese dentária necessária para } \\
\text { substituir os dentes perdidos após uma } \\
\text { ressecção faz parte do planejamento } \\
\text { cirúrgico virtual e da simulação da } \\
\text { ressecção maxilar ou mandibular e do } \\
\text { procedimento de reconstrução. Os } \\
\text { implantes podem ser colocados no } \\
\text { momento da reconstrução da mandíbula, o } \\
\text { que reduzirá o tempo de reabilitação } \\
\text { dentária, aumentará a exposição óssea } \\
\text { ideal durante a colocação do implante e } \\
\text { eliminará a necessidade de oxigênio } \\
\text { hiperbárico após a radioterapia. }\end{array}$ \\
\hline $\begin{array}{l}\text { Ghai, } \\
\text { et al. } \\
2018\end{array}$ & $\begin{array}{l}\text { Demonstrar os usos e a } \\
\text { aplicabilidades da } \\
\text { impressão 3D em cirurgias } \\
\text { craniomaxilofaciais. }\end{array}$ & $\begin{array}{l}\text { A impressão 3D, permite a fabricação sob } \\
\text { demanda e de projetos complexos, ao mesmo } \\
\text { tempo que sejam compatíveis com vários } \\
\text { materiais e fontes de células. As aplicações } \\
\text { variam de modelos anatômicos (principalmente } \\
\text { para planejamento cirúrgico) para guias cirúrgicos } \\
\text { e implantes. }\end{array}$ & $\begin{array}{l}\text { A tecnologia de impressão 3D está sendo } \\
\text { usada com sucesso para cirurgias de } \\
\text { neoplasias de cabeça e pescoço, } \\
\text { reconstrução mandibular, cirurgias } \\
\text { ortognáticas, para mandibulectomias após } \\
\text { osteorradionecrose, cirurgias de fratura do } \\
\text { assoalho orbital, reconstrução nasal e } \\
\text { cranioplastias. }\end{array}$ & $\begin{array}{l}\text { As principais vantagens da tecnologia de } \\
\text { impressão } 3 \mathrm{D} \text { são o tempo cirúrgico } \\
\text { reduzido, o resultado cirúrgico aprimorado } \\
\text { e a exposição reduzida à radiação. A } \\
\text { empolgação por trás da impressão 3D } \\
\text { continua a aumentar e, esperançosamente, } \\
\text { levará a melhorias na tecnologia e em suas } \\
\text { aplicações cirúrgicas, especialmente na } \\
\text { região craniomaxilofacial. }\end{array}$ \\
\hline $\begin{array}{l}\text { Liu, } \\
\text { Hang- } \\
\text { Hang et }\end{array}$ & $\begin{array}{l}\text { Revisar as aplicações } \\
\text { atuais da cirurgia robótica } \\
\text { em casos de câncer na }\end{array}$ & $\begin{array}{l}\text { A revisão incluiu pesquisas feitas usando o } \\
\text { Cochrane Central Register of Controlled Trials, } \\
\text { MEDLINE, Embase, o China National }\end{array}$ & $\begin{array}{l}\text { O sistema cirúrgico robótico é um } \\
\text { procedimento minimamente invasivo } \\
\text { promissor, mas o desenvolvimento da }\end{array}$ & $\begin{array}{l}\text { Os resultados primários da aplicação da } \\
\text { cirurgia robótica na região da cabeça e } \\
\text { pescoço apresenta um bom controle da }\end{array}$ \\
\hline
\end{tabular}




\begin{tabular}{|c|c|c|c|c|}
\hline 2017 & $\begin{array}{l}\text { região da cabeça e do } \\
\text { pescoço, e destacar os } \\
\text { benefícios e limitações dos } \\
\text { atuais sistemas de cirurgia } \\
\text { robótica. }\end{array}$ & $\begin{array}{l}\text { Knowledge Infrastructure e o China Biology } \\
\text { Medicine. Literaturas cinzentas, como OpenGrey } \\
\text { e Sciencepaper Online também foram inclusos. } \\
\text { Pesquisas manuais também foram conduzidas em } \\
\text { revistas chinesas relevantes, e listas de referência } \\
\text { de artigos relevantes foram revisadas. A } \\
\text { linguagem foi restringida a chinês e inglês. No } \\
\text { total foram encontrados } 503 \text { estudos, dos quais } \\
119 \text { eram sobre a aplicação da cirurgia robótica na } \\
\text { região da cabeça e pescoço. }\end{array}$ & $\begin{array}{l}\text { cirurgia robótica continua em um estágio } \\
\text { muito inicial. Ainda são necessárias mais } \\
\text { pesquisas antes de sua ampla aplicação na } \\
\text { cirurgia maxilofacial para neoplasias de } \\
\text { cabeça e pescoço em doenças não } \\
\text { malignas. Os estudos disponíveis } \\
\text { indicaram excelentes resultados em termos } \\
\text { de morbidade cirúrgica, controle } \\
\text { oncológico, e recuperação funcional de } \\
\text { pacientes de tumor de cabeça e pescoço } \\
\text { tratados com sistemas cirúrgicos robóticos. }\end{array}$ & $\begin{array}{l}\text { efermidade, rápida recuperação funcional } \\
\text { pós-operatória e baixa morbidade } \\
\text { cirúrgica. Ainda assim, recomendações } \\
\text { definitivas para a aplicação de sistemas } \\
\text { cirúrgicos robóticos no tratamento de } \\
\text { tumores de cabeça e pescoço, fissura labial } \\
\text { e de palato, OSAS e outras condições, } \\
\text { serão necessários mais estudos melhores } \\
\text { desenvolvidos e modificações técnicas nos } \\
\text { robôs cirúrgicos atuais e futuros. }\end{array}$ \\
\hline $\begin{array}{l}\text { Ma, } \\
\text { Qingchua } \\
\text { n et al. } \\
2019\end{array}$ & $\begin{array}{l}\text { Este estudo propôs um } \\
\text { sistema cirúrgico para } \\
\text { conduzir a cirurgia oral e } \\
\text { maxilofacial com a } \\
\text { assistência e vigilância do } \\
\text { cirurgião. }\end{array}$ & $\begin{array}{l}\text { Um módulo de navegação sem marcadores e um } \\
\text { robô de cirurgia oral e maxilofacial compacto foi } \\
\text { perfeitamente integrado nesse sistema. Um } \\
\text { experimento de treino foi conduzido em cinco } \\
\text { modelos de mandíbula impressos } \\
\text { tridimensionalmente para testar a capacidade de } \\
\text { detecção da posição e avaliar a performance } \\
\text { operacional. }\end{array}$ & $\begin{array}{l}\text { O experimento mostrou que esse sistema } \\
\text { conseguiu guiar o robô até finalizara } \\
\text { operação com sucesso independentemente } \\
\text { da posição da mandíbula. A precisão do } \\
\text { software e do hardware são aceitáveis, e } \\
\text { tem potencial para melhorar a performance } \\
\text { no posicionamento mais preciso. }\end{array}$ & $\begin{array}{l}\text { O sistema propôs um novo conceito e uma } \\
\text { solução prática para reduzir os fatores } \\
\text { relacionados a humanos da cirurgia oral e } \\
\text { maxilofacial, podendo mudar o papel do } \\
\text { cirurgião do futuro na sala de operação e } \\
\text { inclusive beneficiar os resultados da } \\
\text { cirurgia oral e maxilofacial. }\end{array}$ \\
\hline $\begin{array}{l}\text { Smith, } \\
\text { Matthew } \\
\text { M et al. } \\
2016\end{array}$ & $\begin{array}{l}\text { Descrever o uso da } \\
\text { cirurgia trans oral robótica } \\
\text { para remover o adenoma } \\
\text { de paratireoide. }\end{array}$ & $\begin{array}{l}\text { Relato sobre uma mulher de } 55 \text { anos que } \\
\text { apresentou hiperparatireoidismo primário, foi } \\
\text { feita uma tomografia } 4 \mathrm{D} \text { computadorizada e uma } \\
\text { varredura Sestamibi da paratireoide que revelou } \\
\text { uma massa no seio piriforme esquerdo. Usando o } \\
\text { sistema robótico Da Vinci, foi feita uma incisão } \\
\text { hipofaríngea com um eletrocautério no seio } \\
\text { piriforme esquerdo para extrair a massa. }\end{array}$ & $\begin{array}{l}\text { A massa ectópica foi removida através da } \\
\text { abordagem trans oral robótica e a patologia } \\
\text { final confirmou adenoma de paratireoide. } \\
\text { O nível de hormônio da paratireoide caiu } \\
\text { de } 135.8 \text { para } 13.3 \mathrm{pg} / \mathrm{ml} 15 \text { minutos } \\
\text { depois da extração da massa. }\end{array}$ & $\begin{array}{l}\text { O adenoma de paratireoide pode se } \\
\text { apresentar de várias áreas do pescoço } \\
\text { diferentes, sendo o seio piriforme uma } \\
\text { localização em potencial. A cirurgia } \\
\text { robótica trans oral confere a vantagem de } \\
\text { não precisar de incisão externa para a } \\
\text { remoção do adenoma de paratireoide } \\
\text { ectópico. }\end{array}$ \\
\hline
\end{tabular}


Tay,

Gerald et

al.

2017

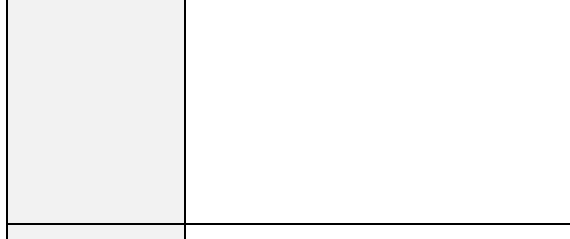

Investigar a viabilidade técnica do uso do TORCS (Cirurgia Robótica Trans

Khan,

Khurram

et al.

2016

Oral de Fenda) para acessar a parede faríngea posterior e o palato para um potencial uso na população com fenda.

\section{Fazer uma revisão de}

Boruman

di, F et al. 2018

como a cirurgia robótica

transoral pode tratar o
Relato sobre um homem de 46 anos com um câncer recorrente na base direita da língua que tinha um trismo de pós-radiação severo. Foi feita uma osteotomia mandibular de linha média sem lip split e isso resultou em respostas positivas na cirurgia. $\mathrm{O}$ paciente foi tratado com uma ressecção feita com TORS na base direita da língua, sem complicações consideráveis.

Todas as possíveis combinações de pacientes com configurações de instrumento robóticos foram feitas com o daVinci Si Surgical System, endoscópios 3D e instrumentos de treinamento para determinar a visualização e 0 acesso cirúrgico ao palato e à faringe posterior em um manequim pediátrico de vias aéreas. E para simular a cirurgia da parede faríngea posterior, foi feita uma faringoplastia de Hynes completamente assistida por robô usando instrumentos de treinamento. câncer no espaço orofaríngeo.
Reuniu relatos da literatura atual que comparam os resultados oncológicos da quimiorradioterapia com o TORS (Cirurgia robótica transoral) para o ratamento de OPSCC (Carcinoma de célula escamosa de orofaringe).
A utilização da osteotomia mandibular de linha média permitiu uma melhor exposição para o uso do TORS em um paciente com uma abertura de boca limitada por causa de trismo de pósradiação. Hemorragia pós-operatória e desconhecimento da medida mais apropriada para diminuir os riscos das catastróficas consequências disso continuam sendo um problema considerável.

Os usos ainda não relatados da cirurgia de TORCS é tecnicamente viável na população pediátrica com fissura. Eles preveem uma pequena curva de aprendizagem devido à instrumentação intuitiva, dissecção mais fácil e melhora da ergonomia para o cirurgião.

\begin{tabular}{|c|c|}
\hline $\begin{array}{l}\text { TORS é uma abordagem cirúrgica } \\
\text { alternativa para tratar tumores de } \\
\text { orofaringe recorrentes e tem resultados } \\
\text { funcionais melhores do que abordagens } \\
\text { cirúrgicas tradicionais. A perda de sangue }\end{array}$ & $\begin{array}{l}\text { TORS permite uma desintensificação do } \\
\text { tratamento de carcinoma orofaríngeo em } \\
\text { estado inicial e evita as toxinas imediatas e } \\
\text { tardias associadas } \\
\text { radioterapia/quimioterapia. Reduz a }\end{array}$ \\
\hline
\end{tabular}

melhorar consideravelmente a capacidade de o cirurgião realizar procedimentos difíceis de palato e faringe posterior em pacientes selecionados com acesso limitado, assim como estabelecer a base para novas técnicas em potencial 


\begin{tabular}{|c|c|c|c|c|}
\hline & & & $\begin{array}{l}\text { intraoperatória, tempo de recuperação pós- } \\
\text { operatória e internação hospitalar são } \\
\text { menores em pacientes que passaram pela } \\
\text { cirurgia robótica. Além disso, pode ser } \\
\text { usado como adjunto às técnicas } \\
\text { convencionais de reconstrução de } \\
\text { orofaringe, e se apresentou viável para } \\
\text { acessar a base da língua, vem sendo usado } \\
\text { no tratamento como OSAHS (Síndrome de } \\
\text { apneia-hipopneia do sono). }\end{array}$ & $\begin{array}{l}\text { duração da internação hospitalar quando } \\
\text { comparada à mandibulotomia labial, o } \\
\text { TORS é considerado uma alternativa } \\
\text { segura, efetiva e potencialmente } \\
\text { econômica. Além disso, o índice de } \\
\text { apneia-hipopneia em pacientes com } \\
\text { OSAHS pode ser significativamente } \\
\text { reduzido com baixa morbidade e uma } \\
\text { internação hospitalar pequena. }\end{array}$ \\
\hline $\begin{array}{l}\text { Kim, Jae } \\
\text { Young et } \\
\text { al. } \\
2016\end{array}$ & $\begin{array}{l}\text { Enfatizar a necessidade de } \\
\text { VSP na era da cirurgia } \\
\text { robótica. }\end{array}$ & $\begin{array}{l}\text { Esse relato apresenta, baseado na experiência dos } \\
\text { autores, as imperfeições da cirurgia sem VSP } \\
\text { (Planejamento cirúrgico virtual) quando } \\
\text { combinado com cirurgia robótica usando incisão } \\
\text { facelift modificada, enfatizando a necessidade de } \\
\text { VSP na era da cirurgia robótica. }\end{array}$ & $\begin{array}{l}\text { Duas pacientes foram encaminhadas do } \\
\text { departamento de otorrinolaringologia para } \\
\text { uma mandibulectomia e reconstrução de } \\
\text { mandíbula. O paciente usando o VSP teve } \\
\text { um resultado de sucesso com a mandíbula } \\
\text { reconstruída simetricamente. É possível } \\
\text { identificar, em uma vista panorâmica, flap } \\
\text { de sucesso e remodelamento ósseo nos } 5 \\
\text { meses seguintes. Infelizmente, o flap da } \\
\text { paciente sem VSP falhou. }\end{array}$ & $\begin{array}{l}\text { VSP e cirurgia robótica, juntos, permitem } \\
\text { um procedimento de bom custo-benefício } \\
\text { e menor tempo de operação se comparado } \\
\text { ao planejamento tradicional, ele pode } \\
\text { estabelecer e aplicar um plano de } \\
\text { tratamento ideal para o paciente, além de } \\
\text { delimitar o campo cirúrgico ao identificar } \\
\text { a margem reduzindo a necessidade de } \\
\text { determinar a margem cirúrgica } \\
\text { frequentemente. }\end{array}$ \\
\hline $\begin{array}{l}\text { Shen, } \\
\text { Zong- } \\
\text { Shan et } \\
\text { al. } \\
2017\end{array}$ & $\begin{array}{l}\text { Comparar as indicações, } \\
\text { abordagens cirúrgicas, e } \\
\text { vantagens e desvantagens } \\
\text { do SND } \quad \text { (Dissecção } \\
\text { seletiva de pescoço) } \\
\text { convencional, } \\
\text { endoscopicamente }\end{array}$ & $\begin{array}{l}\text { Foi feita uma pesquisa sistemática no MEDLINE } \\
\text { (PubMed), Cochrane, ClinicalTrials. gov, e } \\
\text { EMBASE entre janeiro de } 2000 \text { e junho de } 2016 \text {, } \\
\text { com os descritores: “endoscopically assisted neck } \\
\text { dissection,"“robot-assisted neck dissection,,"“neck } \\
\text { dissection," and "minimal invasive." Apenas } \\
\text { artigos associados com dissecção de pescoço }\end{array}$ & $\begin{array}{l}\text { Pacientes com obesidade podem apresentar } \\
\text { maiores desafios para fazer a dissecção via } \\
\text { incisão minimamente invasiva. Se um } \\
\begin{array}{llll}\text { paciente tem histórico de cirurgia } & \text { de } \\
\text { pescoço ou irradiação, a } & \text { alteração da } \\
\text { estrutura anatômica } & \text { dificulta } & \text { a } \\
\text { performance do cirurgião via incisão }\end{array}\end{array}$ & $\begin{array}{l}\text { O SND mostrou-se um procedimento } \\
\text { adequado para OSCC em estágio inicial. } \\
\text { Contudo, não existem dados controlados } \\
\text { randomizados comparando os resultados } \\
\text { do SND endoscopicamente assistido, SND } \\
\text { assistido por robô, e SND convencional, } \\
\text { logo, estudos multicêntricos futuros sobre }\end{array}$ \\
\hline
\end{tabular}




\begin{tabular}{|c|c|c|c|c|}
\hline & $\begin{array}{l}\text { assistida, e SND assistida } \\
\text { por robô para fornecer aos } \\
\text { cirurgiões meios de } \\
\text { escolher a melhor dessas } \\
\text { técnicas para o tratamento } \\
\text { de OSCC (Carcinomas de } \\
\text { Células Escamosas Orais) } \\
\text { em estágio inicial. }\end{array}$ & $\begin{array}{l}\text { endoscopicamente assistida ou assistida por robô } \\
\text { foram incluídas. }\end{array}$ & $\begin{array}{l}\text { pequena. O tumor precisa estar em estágio } \\
\text { inicial, e SND minimamente invasivo é o } \\
\text { mais apropriado para pacientes sem } \\
\text { metástase de nódulos linfáticos. Contudo, } \\
\text { se a dissecção terapêutica de pescoço for } \\
\text { necessária, SND minimamente invasivo } \\
\text { não é adequado. }\end{array}$ & $\begin{array}{l}\text { essas abordagens minimamente invasivas } \\
\text { serão necessários para acumular dados e } \\
\text { estabelecer uma segurança oncológica de } \\
\text { longo prazo. }\end{array}$ \\
\hline $\begin{array}{l}\text { Chao, } \\
\text { Albert H } \\
\text { et al. } \\
2016\end{array}$ & $\begin{array}{l}\text { Investigar uma abordagem } \\
\text { para reconstrução da } \\
\text { mandíbula usando técnica } \\
\text { de retalho da fíbula, no } \\
\text { qual o erro humano pode } \\
\text { ser evitado através de } \\
\text { osteomias } \\
\text { programadas } \\
\text { roboticamente. }\end{array}$ & $\begin{array}{l}\text { Imagens foram adquiridas usando o HRCT (High } \\
\text { Resolution Computed Tomography) da } \\
\text { fíbula/mandíbula, a imagem foi segmentada para } \\
\text { criar uma imagem digital 3D. Usando o CAD, foi } \\
\text { feito um planejamento cirúrgico virtual, traduzido } \\
\text { de planos VSP de osteotomia para um software } \\
\text { robótico, registro da navegação estereotáxica } \\
\text { entre robô e fíbula e execução das osteotomias } \\
\text { pelo robô na fíbula impressa tridimensionalmente. }\end{array}$ & $\begin{array}{l}\text { Navegação estereotáxica (CASPA System, } \\
\text { OrthoMaquet, Rastatt, Germany) foi } \\
\text { implementado para transmitir para o robô } \\
\text { sua posição e orientação em relação à } \\
\text { fíbula e, ao mesmo tempo, prover um } \\
\text { feedback dinâmico em tempo real durante } \\
\text { as osteotomias, para melhorar a precisão. } \\
\text { A reconstrução da mandíbula usando } \\
\text { técnica de retalho da fíbula assistida por } \\
\text { robô é realiza a geração dos planos } \\
\text { osteotômicos através de VSP e transforma } \\
\text { em um guia de corte físico cirúrgico } \\
\text { usando técnicas CAM. Um aparato de } \\
\text { montagem estabilizando a fíbula com } 4 \\
\text { marcações físicas duais foi usado para } \\
\text { delimitar as barreiras físicas ao redor da } \\
\text { fíbula para o robô. }\end{array}$ & $\begin{array}{l}\text { Osteotomias robóticas totalmente pré- } \\
\text { programadas para reconstrução mandibular } \\
\text { com retalho fibroso livre pode atingir um } \\
\text { alto grau de precisão e consistência, pode } \\
\text { ser útil no desenvolvimento de técnicas } \\
\text { para melhorar ainda mais a precisão } \\
\text { cirúrgica. Porém estudos mais } \\
\text { aprofundados ainda são necessários, } \\
\text { incluindo a maneira pela qual um robô } \\
\text { seria integrado em um espaço físico } \\
\text { ocupado por cirurgiões e outros } \\
\text { equipamentos, questões de segurança, } \\
\text { como em relação ao pedículo vascular, e } \\
\text { resultados cirúrgicos que seriam } \\
\text { necessários para justificar seu uso ao } \\
\text { considerar os fatores como custo. }\end{array}$ \\
\hline
\end{tabular}

Fonte: Autores (2020). 


\subsubsection{Tipos e quantidades de artigos}

Foram selecionados, dentre os critérios, 18 artigos científicos. Entre casos clínicos, estudos in vitro, revisões, estudos de viabilidade e ensaios clínicos. Os resultados mostraram que o emprego de robôs como ferramenta se mostrou bem sucedido para diversas aplicações como na implantodontia, em cirurgias minimamente invasivas, no tratamento de câncer de cabeça e pescoço, em cirurgias craniomaxilofaciais e em osteotomias pré-programadas roboticamente. Apesar disso, ainda se faz necessário um maior número de pesquisas clínicas, em especial, randomizadas, com um maior número de pacientes e aplicações nos diversos ramos das cirurgias de cabeça e pescoço para se obter conclusões mais significativas.

\section{Discussão}

A literatura mostra diversas possibilidades para o uso de robôs na cirurgia odontológica, desde o planejamento até a efetuação, segundo autores como o Liu et al (2017). Entretanto, autores como Liu et al (2017) e Kumar et al (2016) apresentam, em seus trabalhos, as limitações desta tecnologia, tais como alto custo, falta de feedback tátil e o período operatório mais longo. Ainda assim, a cirurgia robótica na odontologia demonstra um grande potencial para a melhoria do processo e planejamento cirúrgico, conforme autores como Alfouzan et al (2018), Liu et al (2017) e Gulati et al (2015).

Os estudos como os de Liu et al (2017), Ma et al. (2019) e Shen et al (2017) apresentam inúmeras possibilidades para a aplicação da robótica na cirurgia odontológica, tais como seu uso no tratamento da fissura labiopalatina, no tratamento de fraturas maxilofaciais, na excisão cirúrgica de adenoma de paratireoide ectópico e na dissecção seletiva do pescoço para carcinoma epidermóide oral em estágio inicial. A aplicação da robótica na cirurgia se mostrou eficaz em implantodontia, no tratamento de câncer de cabeça e pescoço, tratamento de neuralgia do trigêmeo e tratamento de pequenas lesões no seio piriforme e osteotomias, de acordo com os estudos de Reddy et al (2015) e Mazerolle et al (2018). Além das aplicações práticas já citadas, Sun et al (2020), Ghai, et al (2018) e Kim et al (2016) também ressaltam a eficácia da robótica no planejamento cirúrgico, através do VSP (Virtual Surgical Planning) e da impressão 3D de modelos anatômicos.

As cirurgias com a presença de robôs são indicadas em diversos tipos de procedimentos na odontologia, principalmente em intervenções complexas que necessitam de uma alta precisão ou de resultados funcionais e estéticos superiores às cirurgias convencionais, ou intervenções nas quais antes o procedimento não era possível, como pode-se ver nas citações de autores como Sun et al. (2020), Alfouzan et al. (2018), Gulati et al. (2015), Reddy et al.(2015) e Kumar et al. (2016). Por essas razões, esses mesmos autores, além de outros como o Borumandi et al. (2018), indicam a aplicação da robótica, por exemplo, na implantodontia, no tratamento do câncer de cabeça e pescoço, no tratamento de neuralgia do trigêmeo, nas cirurgias maxilofaciais, além de outros procedimentos. Eles ressaltam que ocorrem diferenças significativas nos resultados dos procedimentos que utilizam robôs e esses são considerados superiores aos procedimentos nos quais essa tecnologia não está presente.

Autores como Gulati et al. (2015), Reddy et al. (2015) e Mazerolle et al. (2018) são unânimes ao relatarem, em suas pesquisas, que o sistema cirúrgico robótico tem benefícios notáveis na diminuição de complicações, na melhora da qualidade de vida dos pacientes, em resultados estéticos melhores, na diminuição do tempo de recuperação dos pacientes e tem um maior custo-benefício em relação ao planejamento cirúrgico em comparação com cirurgia convencional sem auxílio de ferramentas robóticas.

É importante ressaltar que o alto custo é a principal limitação da utilização de robôs em procedimentos cirúrgicos, de acordo com Liu et al. (2019). Ele cita que isso varia conforme a complexidade das intervenções cirúrgicas e a consequente dificuldade de treinamento de algumas técnicas pela equipe. Outro fator a ser considerado é que as cirurgias realizadas por robôs compartilham os mesmos riscos da cirurgia tradicional, com os mesmos problemas potenciais de infecção, anestesia ou relacionados a qualquer tipo de cirurgia, entretanto, segundo o próprio Liu et al. (2019), há um risco adicional único de 
cirurgias robóticas que deve ser levado em consideração, o risco de falha mecânica.

Os robôs na odontologia são destaques por apresentarem um bom controle em relação a sua precisão, rápida recuperação funcional pós-operatória e baixa morbidade cirúrgica, como pode ser observado nas citações de autores como Liu et al (2017), Borumandi et al. (2018) e Kim et al (2016). Além disso, alguns artigos, como o de Borumandi et al (2018), relataram que os procedimentos assistidos por robôs reduzem a duração da internação hospitalar, quando comparadas ao tratamento comum, sendo considerada uma alternativa segura, efetiva e potencialmente econômica, sendo o seu custo-benefício evidente.

Este estudo se trata de uma revisão integrativa, nele foram analisados estudos relevantes sobre a aplicação da robótica na cirurgia odontológica, fazendo uma síntese dos conhecimentos relacionados a esse assunto, indicando as principais vantagens, desvantagens, dificuldades e necessidades de estudo com relação ao assunto referido, sendo, assim, um suporte em potencial para profissionais de saúde. Este estudo se torna relevante porque foram encontrados 18 artigos que foram selecionados pelo uso dos descritores "Robotic Surgical Procedures, dentistry, oral surgery" e que posteriormente foram pesquisados nas bases de dados CAPES, PubMed e MEDLINE, que são referências mundiais. Essa busca resultou em artigos compatíveis, que foram selecionados, e em incompatíveis com o objetivos dos autores, e estes foram excluídos. O uso de "And" entre os descritores foi essencial para afunilar os resultados e obter uma busca precisa e artigos relacionados aos objetivos. Por sua vez, ao utilizarmos os bancos de dados para a pesquisa, foram encontradas certas dificuldades para a obtenção dos artigos na íntegra, pois, a maioria deles é publicado em revistas/anais pagos, mas esse problema foi resolvido com a ajuda da Universidade de Pernambuco e da professora orientadora. A pesquisa resultou em uma síntese de dados e recomendações que tem como propósito colaborar com a comunidade acadêmica e profissionais cirurgiões-dentistas ao apresentar resultados de pesquisas com fontes seguras e com o uso de descritores cadastrados em bancos de dados e plataformas seguras com credibilidade acadêmica.

Quanto às limitações à aplicação dessa tecnologia, as principais são o custo relativamente alto, se comparada à cirurgia convencional, tempo de operação maior e a necessidade de maior capacitação de profissionais para lidar com essa tecnologia, como ressaltado por autores como Kumar et al (2016). Além disso, os estudos de autores como Ma et al (2019); Shen et al (2017) e Liu et al (2017) são unânimes ao constatar que, apesar de sua aplicabilidade ser muito promissora na cirurgia odontológica, ainda são necessários ajustes para que a aplicação de sistemas cirúrgicos robóticos se tornem rotina, ou seja, ainda serão necessários mais estudos e modificações técnicas nos robôs cirúrgicos atuais e futuros, para que os procedimentos se tornem mais efetivos e seguros.

\section{Considerações Finais}

Diante do exposto, podemos afirmar que a literatura converge ao considerar a aplicação da robótica em procedimentos cirúrgicos odontológicos uma combinação de grande potencial com relação à segurança, precisão, melhor recuperação funcional pós-operatória e melhores resultados estéticos.

Entretanto, observamos que ainda há necessidade de novos estudos para que a utilização de robôs na odontologia possa ser recomendada definitivamente nos diversos tipos de procedimentos possíveis na odontologia e para avaliar o grau de sucesso posterior desse tipo de intervenção, além do desenvolvimento de tecnologias mais avançadas para que essas aplicações alcancem resultados melhores e mais precisos.

Esse desenvolvimento tecnológico e aprimoramento dessas ferramentas têm como tendência solucionar a maior limitação observada pelos autores, que é o alto custo tanto para os profissionais, clínicas e hospitais fornecerem essa tecnologia quanto para os pacientes quando têm que arcar com os custos desses procedimentos.

Além das aplicações atuais da cirurgia robótica na odontologia já destacadas pelos autores, como em implantodontia e 
no tratamento de câncer de cabeça e pescoço, ainda existem outras linhas de pesquisa que podem ser desenvolvidas para se estudar outras aplicações, como a aplicação dessa tecnologia para osteotomias robóticas totalmente pré-programadas para reconstrução mandibular com retalho fibroso livre mencionada nos estudos de Chao et al (2016).

\section{Referências}

Alfouzan A. F. (2018). Review of surgical resection and reconstruction in head and neck cancer. Traditional versus current concepts. Saudi medical journal, 39(10), 971-980. 10.15537/smj.2018.10.22887

Borumandi, F., \& Cascarini, L. (2018). Robotics in oral and maxillofacial surgery. Annals of the Royal College of Surgeons of England, 100(6), 16-18. $10.1308 /$ rcsann.supp 1.16

Chao, A. H., Weimer, K., Raczkowsky, J., Zhang, Y., Kunze, M., Cody, D., Selber, J. C., Hanasono, M. M., \& Skoracki, R. J. (2016). Pre-programmed robotic osteotomies for fibula free flap mandible reconstruction: A preclinical investigation. Microsurgery, 36(3), 246. 10.1002/micr.30013

Ghai, S., Sharma, Y., Jain, N., Satpathy, M., \& Pillai, A. K. (2018). Use of 3-D printing technologies in craniomaxillofacial surgery: a review. Oral and maxillofacial surgery, 22(3), 249-259. 10.1007/s10006-018-0704-z

Gulati, M., Anand, V., Salaria, S. K., Jain, N., \& Gupta, S. (2015). Computerized implant-dentistry: Advances toward automation. J Indian Soc Periodontol 2015; 19:5-10. 10.4103/0972-124X.145781

Khan, K., Dobbs, T., Swan, M. C., Weinstein, G. S., \& Goodacre, T. E. (2016). Trans-oral robotic cleft surgery (TORCS) for palate and posterior pharyngeal wall reconstruction: A feasibility study. Journal of plastic, reconstructive \& aesthetic surgery: JPRAS, 69(1), 97-100. 10.1016/j.bjps.2015.08.020

Kim, J. Y., Kim, W. S., Choi, E. C., \& Nam, W. (2016). The Role of Virtual Surgical Planning in the Era of Robotic Surgery. Yonsei Med J. 2016;57(1):265268. 10.3349/ymj.2016.57.1.265

Kumar, A., Yadav, N., Singh, S., \& Chauhan, N. (2016). Minimally invasive (endoscopic-computer assisted) surgery: Technique and review. Annals of maxillofacial surgery, 6(2), 159-164. 10.4103/2231-0746.200348

Ling, D. C., Chapman, B. V., Kim, J., Choby, G. W., Kabolizadeh, P., Clump, D. A., Ferris, R. L., Kim, S., Beriwal, S., Heron, D. E., \& Duvvuri, U. (2016). Oncologic outcomes and patient-reported quality of life in patients with oropharyngeal squamous cell carcinoma treated with definitive transoral robotic surgery versus definitive chemoradiation. Oral oncology, 61, 41-46. 10.1016/j.oraloncology.2016.08.004

Liu, H. H., Li, L. J., Shi, B., Xu, C. W., \& Luo, E. (2017). Robotic surgical systems in maxillofacial surgery: a review. International journal of oral science, 9(2), 63-73. 10.1038/ijos.2017.24

Liu, H., Wang, Y., Wu, C., Sun, X., Li, L., Li, C., \& Luo, E. (2019). Robotic compared with open operations for cancers of the head and neck: a systematic review and meta-analysis. The British journal of oral \& maxillofacial surgery, 57(10), 967-976. 10.1016/j.bjoms.2019.08.023

Ma, Q., Kobayashi, E., \& Wang, J. (2019). Development and preliminary evaluation of an autonomous surgical system for oral and maxillofacial surgery. Int J Med Robot. 2019; 15(4):e1997. 10.1002/rcs.1997

Mazerolle, P., Philouze, P., Garrel, R., Aubry, K., Morinière, S., El Bedoui, S., Ton Van, J., Ferron, C., Malard, O., Jegoux, F., Berard, E., \& Vergez, S. (2018). Oncological and functional outcomes of trans-oral robotic surgery for pyriform sinus carcinoma: A French GETTEC group study. Oral oncology, 86, 165-170. 10.1016/j.oraloncology.2018.09.014

Pereira, A. S., Shitsuka, D. M., Parreira, F. J. \& Shitsuka, R. et al. (2018). Metodologia da pesquisa científica. https://repositorio.ufsm.br/bitstream/handle/1/15824/Lic_Computacao_Metodologia-Pesquisa-Cientifica.pdf?sequence=1

Reddy, E. P., Sinha, S., Chandra, S., \& Chandra, S. (2015). CyberKnife radiosurgery: Precision without incision. J Indian Acad Oral Med Radiol 2015; 27:725. 10.4103/0972-1363.167088

Shen, Z. S., Li, J. S., Chen, W. L., \& Fan, S. (2017). The Latest Advancements in Selective Neck Dissection for Early Stage Oral Squamous Cell Carcinoma. Current treatment options in oncology, 18(5), 31. 10.1007/s11864-017-0471-3

Smith, M. M., Young, W. G., Carlin, A. M., \& Ghanem, T. A. (2016). Trans-oral robotic surgical excision of an ectopic parathyroid adenoma. J Robot Surg. 2016;10(1):73-75. 10.1007/s11701-015-0545-9

Sun, T.-M., Lee, H.-E., \& Lan, T.-H. (2020). Comparing Accuracy of Implant Installation with a Navigation System (NS), a Laboratory Guide (LG), NS with LG, and Freehand Drilling. International Journal of Environmental Research and Public Health, 17(6), 2107. 10.3390/ijerph17062107

Tay, G., Ferrell, J., \& Andersen, P. (2017). Use of a midline mandibular osteotomy to improve surgical access for transoral robotic resection of the base of tongue in a patient with trismus. Head and Neck, 39(9), E92-E95. 10.1002/hed.24851 